\title{
Frequência de problemas de equilíbrio nos cascos de cavalos crioulos em treinamento
}

Leandro Schwarcke do CANTO $^{1}$

Flavio Desessards de La CORTE $^{1}$

Karin Erica BRASS

Marcelo Dellazzana

RIBEIRO $^{1}$

Correspondência para:

LEANDRO SCHWARCKE DO CANTO

Rua Dr. Bozano 947 ap. 301

97015-003 - Santa Maria-RS

leandrodocanto@brturbo.com

Recebido para publicação: 25/08/2004 Aprovado para publicação: 01/06/2005

1 - Departamento de Clínica de Grandes Animais da Faculdade de Medicina Veterinária da Universidade Federal de Santa Maria, Santa Maria - RS

\section{Resumo}

O desequilíbrio dos cascos como fator causador de claudicação em cavalos atletas é bastante documentado na literatura e pode ser dividido em geométrico, dinâmico e natural. O presente estudo verificou o equilíbrio geométrico nos cascos de 97 cavalos crioulos adultos, em treinamento, em 16 estabelecimentos, para determinar a incidência de alterações nos cascos. Foram registradas nove medidas nos cascos dos membros anteriores: ângulo da pinça, circunferência na banda coronária, comprimento lateral e medial dos talões e quartos, comprimento da pinça, comprimento e largura da ranilha. As mensurações permitiram a identificação das seguintes alterações: 85 dos 97 cavalos $(87.62 \%)$ apresentaram talões contraídos, $48(49.48 \%)$ desequilíbrio médio-lateral, 23 (23.71\%) ângulos dos cascos contralaterais diferentes e $11(11.34 \%)$ tinham o eixo quebrado para trás. A incidência de ângulos dos cascos contralaterais diferentes variou entre os cinco grupos de idade $(\mathrm{p}<0.05)$. O ângulo do casco variou entre $45^{\circ}$ e $58^{\circ}\left(52.71 \pm 0.15^{\circ}\right)$. O comprimento médio da pinça foi $8.7 \pm 0.6 \mathrm{~cm}$ e foi considerado excessivo para cavalos com $411.4 \pm$ $34.80 \mathrm{~kg}$ de peso. A grande freqüência de alterações de casco nesta população de cavalos sugere que as práticas de casqueamento e ferrageamento adotadas devem ser revistas e melhoradas.

\section{Introdução}

Os cavalos de competição de alto nível, que desempenham suas atividades próximas ao limite, podem ter a sua performance afetada negativamente devido a alterações no aparelho locomotor tais como uma má conformação dos cascos. $\mathrm{O}$ desequilíbrio dos cascos, ou seja, a presença de assimetrias nos cascos, é um dos fatores mais importantes na origem de claudicações em eqüinos. Ele é resultado de defeitos de conformação nos membros ou casqueamento incorreto. Isto gera importantes perdas econômicas quando são considerados os gastos com treinamento, atendimento veterinário, tempo de treinamento perdido, alimentação e investimentos na seleção dos animais.

As extremidades distais dos membros do cavalo funcionam como um distribuidor de forças que resultam da interação entre o cavalo e a superfície por onde ele caminha. No animal com conformação ideal, o centro de gravidade do casco é o mesmo do membro.

As causas mais comuns de claudicação em eqüinos estão relacionadas com alterações nos cascos dos membros anteriores. $\mathrm{O}$ casqueamento e o ferrageamento agem sobre o casco e membro proximal, mais especificamente na distribuição do peso sobre o membro. Isto determinará a distribuição medial/lateral das forças sobre o esqueleto, assim como as forças aplicadas sobre os ligamentos e tendões ${ }^{1}$.

Quando as forças não são uniformes, o suprimento sangüíneo é comprometido resultando em remodelação da falange distal, assim como, distorção da simetria do casco ${ }^{2}$.Portanto, é fundamental que o casque- 
amento e ferrageamento estabeleçam este equilíbrio, caso contrário, a distribuição do peso e das forças na parte distal do membro será alterada.

O estojo córneo é um elemento complexo e muito importante no mecanismo de absorção da concussão. Alterações anatômicas como: "cascos muito pequenos para o tamanho corporal do cavalo" e práticas inadequadas de ferrageamento que prejudicam a capacidade de absorção podem aumentar a ocorrência de claudicação ${ }^{1}$.

Seis alterações de equilíbrio no casco relacionadas à claudicação foram descritas 3: ângulos de pinça e da quartela diferentes, talões contraídos, desequilíbrio médio-lateral, ângulos das pinças e dos talões desiguais, ângulos das pinças dos membros esquerdo e direito diferentes, cascos muito pequenos em relação ao tamanho do animal.

Em 1987, pesquisadores concluíram que o casqueamento e ferrageamento corretos podem melhorar a performance e reduzir a incidência de lesões músculoesqueléticas em cavalos Puro Sangue de Corrida $^{4}$. O equilíbrio crânio-caudal e médio-lateral são essenciais na prevenção de lesões, especialmente nos membros anteriores dos cavalos Puro Sangue de Corrida. Lesões como desmites, tendinites, alterações degenerativas das articulações interfalangeanas e metacarpo-falangeanas, fraturas de primeira falange, sesamoidites, fraturas de sesamóide e doença do navicular estão associadas com ângulos de casco incorretos. Embora o desequilíbrio dos cascos esteja presente em muitos cavalos, alguns indivíduos são capazes de tolerar estas alterações e os problemas aparecem com o avanço da idade do animal. Na maioria dos casos, este desequilíbrio resulta em claudicação. Um casco desequilibrado pode ser a causa primária de dor através do comprometimento do mecanismo de absorção da concussão ou ainda uma causa indireta de dor localizada em uma região mais proximal do membro ${ }^{5}$.

Este trabalho foi desenvolvido com o objetivo de fazer um levantamento da ocorrência de alterações de equilíbrio nos cascos de cavalos Crioulos em treinamento para provas funcionais no Estado do Rio Grande do Sul.

\section{Materiais e Métodos}

Os cascos dos membros anteriores de 97 cavalos Crioulos adultos (60 machos e 37 fêmeas) foram mensurados. A idade dos animais variou de 2 a 13 anos com idade média de 5,42 $\pm 2,25$ anos, e o peso variou de 270 a $486 \mathrm{~kg}$ com média de 411,39 \pm $34,8 \mathrm{~kg}$, todos estavam em treinamento para provas funcionais. As mensurações foram feitas conforme metodologia estabelecida por Turner ${ }^{1,3}$.

Foram tomadas as seguintes medidas (em centímetros) com a utilização de uma fita métrica: comprimento medial e lateral dos talões, comprimento da pinça, comprimento medial e lateral nos quartos. Também foi mensurado o comprimento e a largura da ranilha. A circunferência do casco foi mensurada logo abaixo da coroa e o ângulo da pinça, ângulo formado pela intersecção da face dorsal da parede do casco na região da pinça com o plano horizontal da sola, utilizando um podogoniômetro. Com o uso de uma fita de correlação entre o peso e o perímetro torácico o peso dos cavalos foi estimado. O comprimento da ferradura foi registrado, para comparação com o comprimento de pinça e o alinhamento dos cravos foi observado. A partir destas medidas foram calculadas a relação do peso com área de casco e a relação entre o comprimento e a largura da ranilha. Todas estas medidas foram registradas em uma ficha de serviço para cada cavalo.

Com os dados colhidos, os cavalos foram divididos em cinco grupos de acordo com a idade: G 1 (2 a 3,5 anos) 19,6\% dos animais, $G 2$ (4anos) 22,7\% dos animais, G 3 (5 anos) 15,5\% dos animais, G 4 (6 anos) 20,6\% dos animais e G 5 ( 7 a 13 anos) 21,5\% dos animais. Para verificar a incidência do 
desequilíbrio nos cascos dos cavalos Crioulos e também para identificar as principais causas do problema, foram efetuadas medidas descritivas de tendência central (média) e de dispersão (desvio padrão) para cada uma das variáveis estudadas, e utilizada a análise de variância em um delineamento inteiramente casualizado. Diferenças foram estabelecidas a $\mathrm{p}<0,05$.

\section{Resultados e Discussão}

As alterações mais observadas nos cascos dos cavalos examinados foram: talões contraídos, desequilíbrio médio-lateral, ângulo da pinça diferente entre membros opostos, ângulo da pinça e quartela desiguais e comprimento excessivo da pinça. Todos animais examinados apresentaram pelo menos um tipo de alteração na conformação dos cascos dos membros anteriores.

Em $85(87,62 \%)$ cavalos a largura da ranilha foi inferior a $67 \%$ do seu comprimento o que é caracterizado como talões contraídos ${ }^{1}$. Essa alta freqüência pode estar relacionada com o comprimento médio de pinça encontrado que foi de 8,7 $\pm 0,6 \mathrm{~cm}$ (Figura 1) e foi considerado excessivo para cavalos com 411,40 $\pm 34,80$ $\mathrm{kg}$ de peso.

O comprimento da pinça é medido a partir da coroa do casco até a ponta da pinça. As medidas esperadas estão relacionadas com o peso do animal devendo levar em consideração a posição da falange distal em relação ao estojo córneo, o formato do casco e o uso do animal. Em cavalos com 360 a $400 \mathrm{~kg}$ a pinça deve ter um comprimento de até $7,6 \mathrm{~cm}$, em cavalos com 425 a $475 \mathrm{~kg}$ até $8,25 \mathrm{~cm}$ e cavalos de 525 a $575 \mathrm{~kg}$ até $8,9 \mathrm{~cm}$. A pinça excessivamente curta pode estar relacionada com fragilidade da muralha ou com o aparo excessivo do casco, predispondo sola e ranilha a traumas e o córium à formação de hematomas. O comprimento excessivo das pinças leva ao estreitamento dos cascos, torna a sola plana e leva à contração dos talões, favorecendo infecções anaeróbicas nos sulcos laterais e central da ranilha ${ }^{6}$.

Levando em consideração que em nenhum animal foi encontrada uma relação entre comprimento de pinça e comprimento de talões inferior a 3:1 podemos concluir que a maioria dos animais apresenta cascos muito compridos como um todo e, provavelmente, solas muito espessas o que além de limitar a capacidade de expansão do casco, também desloca a ferradura cranialmente, não proporcionando suporte adequado aos talões e predispondo à ocorrência de talões contraídos, entre outros problemas. Essa freqüência foi maior que a encontrada por Nicoletti et $\mathrm{al}^{7}$ que fez mensurações em 68 eqüinos, de diversas raças, desses animais 30 apresentaram os talões contraídos, em pelo menos um casco, representando $44,11 \%$ do total. Os autores também constataram que o comprimento médio das pinças dos animais examinados estava acima do esperado em relação ao peso dos animais, encontrando $8,2 \mathrm{~cm}$ para animais com 360 a $400 \mathrm{~kg}, 8,7 \mathrm{~cm}$ para animais com 425 a $475 \mathrm{~kg}$ e 9,1 para animais com 525 a $575 \mathrm{~kg}$. Portanto não é apenas na raça Crioula que encontramos alterações como estas.

Existem fatores predisponentes e determinantes que levam à ocorrência de talões contraídos; os fatores que predispõe são aqueles relacionados com a raça e conformação do animal. Acredita-se que devido a fatores genéticos a Pura Raça Espanhola apresenta grande incidência dessa alteração. Entretanto, nessa raça tem-se observado grande incidência somente nos animais que são estabulados e ferrados. Entre os fatores determinantes estão as causas mecânicas (ferrageamento), físicas (diminuição da elasticidade) e dinâmicas (todos os fatores causadores de dor e transtorno funcional) ${ }^{8}$.

Segundo Turner ${ }^{1}$, uma diferença entre o comprimento medial e lateral dos talões de $0,5 \mathrm{~cm}$ ou mais é considerada significativa e indica desequilíbrio médio-lateral do casco. Dos animais avaliados neste trabalho 48 $(49,48 \%)$ apresentaram uma diferença igual 
Comprimento dos cascos

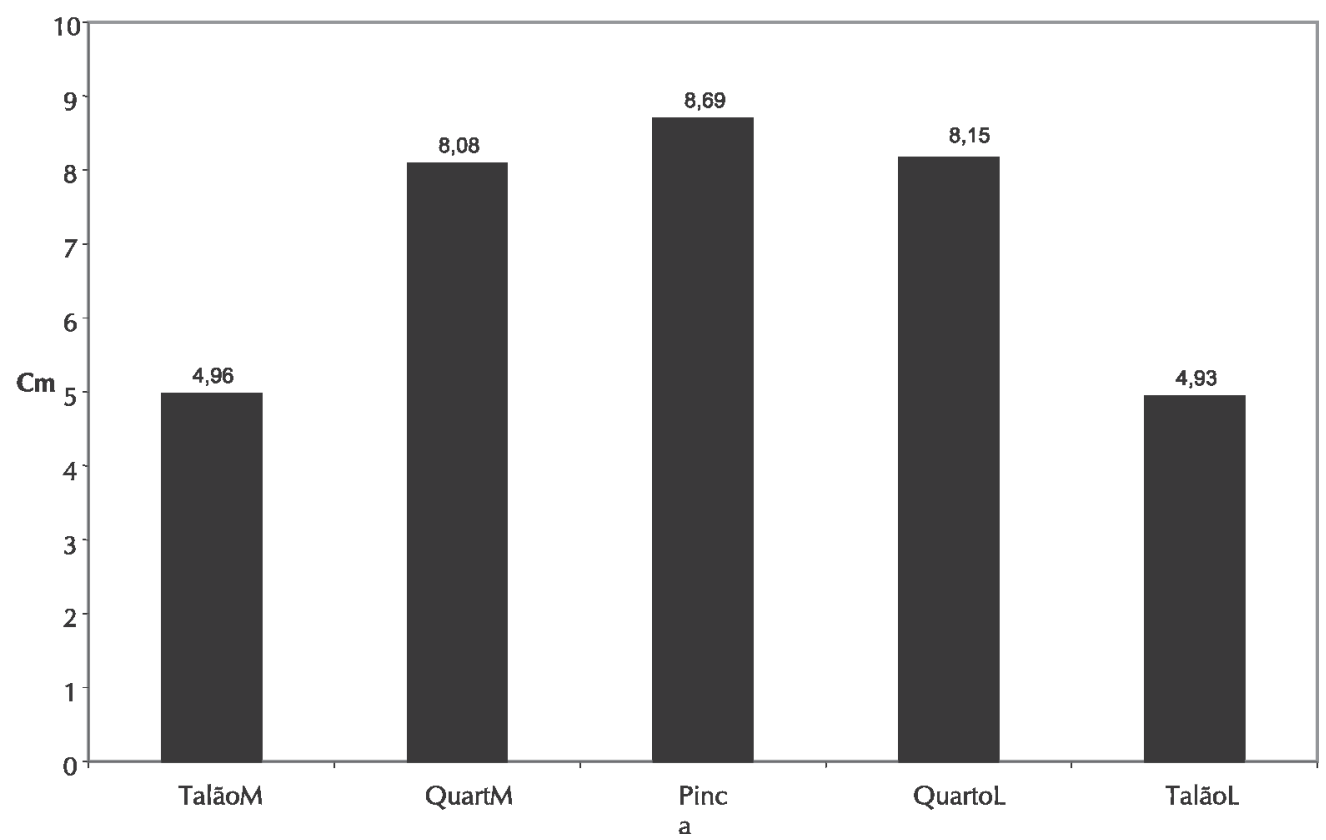

Figura 1 - Médias do comprimento da pinça, quartos lateral e medial e talões lateral e medial

Porcentagem de alteraçōes por grupo de idade

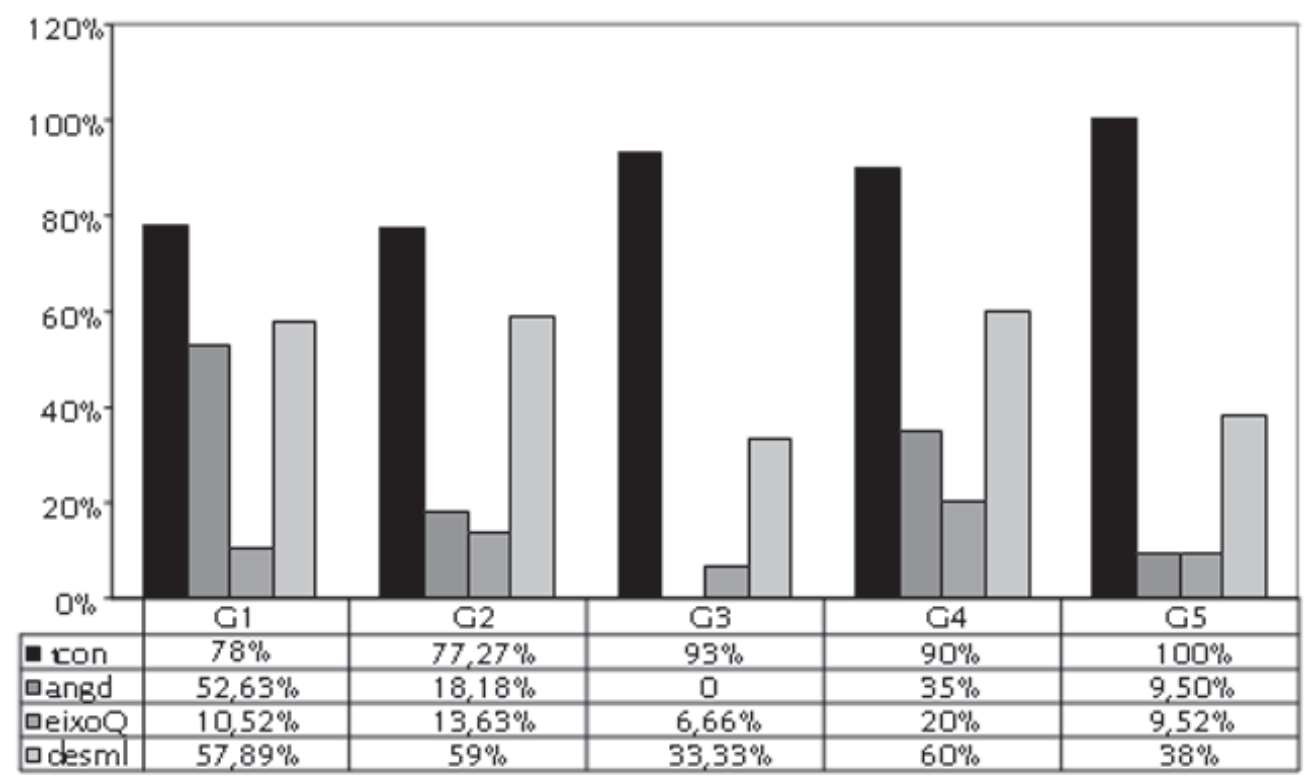

grupos

Figura 2 - Distribuição das alterações: talões contraídos (tcon), ângulo dos membros contra-laterais diferentes (angd), eixo quebrado (eixo Q) e desequilíbrio médio-lateral conforme o grupo etário dos animais onde, G1 (2 a 3,5 anos), G2 (4 anos), G3 (5 anos), G4 (6 anos) e G5 (7 a 13 anos) 
Percentagem de alteraçōes em relaçāo ao total de animais obsenvados

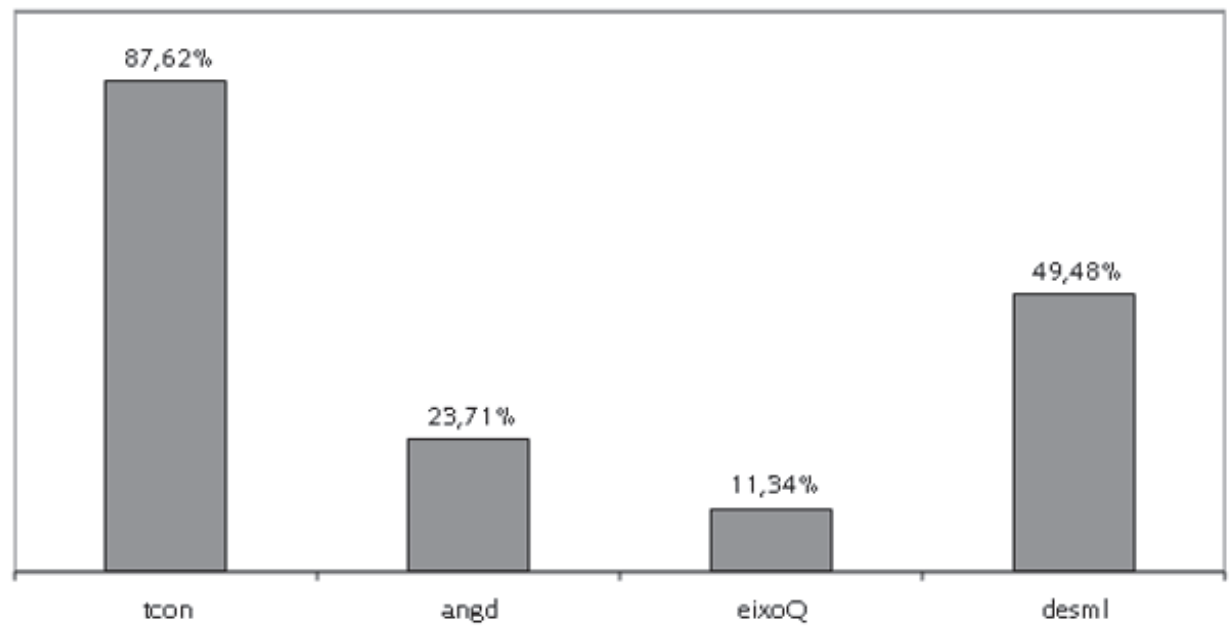

Figura 3 - Porcentagem das principais alterações de cascos encontradas na população de cavalos crioulos em treinamento

ou superior a $0,5 \mathrm{~cm}$ entre o comprimento medial e lateral dos talões em pelo menos um dos membros Figura 3. Essa alteração tem sido apontada como causa de claudicação, apesar de poder ser encontrada em animais que não apresentam claudicação. Acredita-se que a contínua má distribuição da força de impacto com o solo e o aumento da carga sobre um dos talões pode predispor o casco à dor, hematomas subsolares, rachaduras nos quartos e talões, fraturas de casco, osteíte pedal, fissuras na base da ranilha e infecções anaeróbicas ${ }^{9}$.

O eixo pinça quartela é um dos parâmetros mais importantes a serem seguidos pelo ferrador, e é a maneira mais fácil de tentar manter o casco corretamente alinhado com a coluna óssea. A superfície dorsal do casco deve ser paralela à superfície dorsal da quartela. Em 11 animais (11,34\%) foram observadas diferenças entre o ângulo da quartela e o ângulo da pinça. Esta porcentagem é inferior a encontrada por Kobluk et al. ${ }^{4}$, que constatou a alteração em $52 \%$ dos cavalos por ele examinados. Devese considerar que este autor examinou somente cavalos Puro Sangue de Corrida e esta alteração é considerada comum nessa raça. O eixo quebrado para trás, ângulo de pinça menor que o ângulo de quartela, muitas vezes é acompanhado de talões colapsados e pinças compridas.

O'Grady e Poupard ${ }^{9}$ citam que o eixo quebrado para trás leva a extensão da articulação interfalangeana, aumento de tensão do tendão flexor digital profundo e faz com que o casco aterrisse primeiro com a pinça. Isto aumenta a tensão das estruturas associadas ao osso navicular. O eixo quebrado para frente por sua vez, leva a uma flexão da articulação interfalangeana, os talões passam a tocar o solo primeiro, aumentando a pressão nos talões o que pode resultar em inflamação das articulações interfalangeanas distais.

A diferença do ângulo da pinça entre os membros contra-laterais foi identificada em 23 animais $(23,71 \%)$. A incidência variou entre os grupos etários $(p<0.05)$. Ela foi maior no $G 1$ (2,5 a 3 anos) representando $52,63 \%$ dos animais desse grupo Figura 2. Os 19 animais deste grupo estavam distribuídos por 12 centros de treinamento. A presença dessa alteração pode ter ocorrido devido a erros de casqueamento e ferrageamento, defeitos de conformação ou claudicação crônica. $\mathrm{O}$ ângulo da pinça variou de $45^{\circ}$ a $58^{\circ}\left(52,71 \pm 0,15^{\circ}\right)$. 
Em 55 animais foi feita a mensuração do comprimento da ferradura. Destes nenhum estava com ferraduras que tivessem duas vezes o comprimento da pinça o que seria considerado adequado ${ }^{10}$. O comprimento da ferradura variou de 9 a $13 \mathrm{~cm}$. O menor comprimento de pinça entre esses animais foi de $7,5 \mathrm{~cm}$ e o maior de $10 \mathrm{~cm}$. Com o comprimento excessivo do casco, todos apresentavam a ferradura deslocada cranialmente sem proporcionar um suporte adequado dos talões o que além de predispor a contração dos talões, diminui a capacidade de absorção da concussão

\section{Conclusões}

A mensuração dos diferentes parâmetros nos cascos de 97 cavalos crioulos em treinamento para provas funcionais no Estado do Rio Grande do Sul permitiu a identificação de uma alta freqüência de alteração de equilibrio nos cascos como: talões contraídos, ângulos dos cascos dos membros contra-laterais diferentes, eixo quebrado e desequilibrio médiolateral. As médias de comprimento de pinça, quartos e talões sugerem que os cascos desses cavalos estão com o comprimento excessivo, o que prejudica o seu funcionamento, assim como pode predispor a outras alterações de equilíbrio. As práticas de casqueamento e ferrageamento empregadas nesses cavalos devem ser revistas, assim como as causas da alta freqüência dessas alterações investigadas. Proprietários, treinadores e veterinários devem ser alertados para a importância dessas alterações de casco e o seu papel na indução de claudicação no cavalo atleta.

\section{Frequency of hoof balance problems in training crioulo horses}

\section{Abstract}

The role of improper hoof balance causing lameness in sport horses is well documented in the literature. Hoof balance can be divided into geometric, dynamic and natural balance. This study assessed the geometric balance of 97 adult Crioulo horses in training at 16 different training centers to determine the incidence of poor hoof conformation. Nine measurements of the hoof were taken: angle at the toe, circumference at the coronary band, lateral and medial quarter and heel length, toe length, frog length and width. From these measurements the following hoof problems could be identified: 85 out of 97 horses $(87.62 \%)$ had contracted heels, $48(49.48 \%)$ had medial to lateral hoof imbalance, $23(23.71 \%)$ had mismatched hoof angles and $11(11.34 \%)$ had a broken back hoof axis. The frequency of mismatched hoof angles was different between the five age groups $(\mathrm{p}<0.05)$. The hoof angle varied from $45^{\circ}$ to $58^{\circ}\left(52.71 \pm 0.15^{\circ}\right)$. The average toe length was $8.7 \pm 0.6 \mathrm{~cm}$ and was considered to be excessive for horses with $411.4 \pm 34.80 \mathrm{Kg}$ body weight. The high frequency of hoof abnormalities in this population of horses suggests that trimming and shoeing practices employed should be reviewed and improved.

\section{Referências}

1 TURNER, T. The use of hoof measurements for the objective assessment of hoof balance. In: AMERICAN ASSOCIATION OF EQUINE PRACTITIONERS ANNUAL CONVENTION, 38; 1992 Orlando. Proceedings... Orlando: AAEP, 1993. p. 389-395.

2 BUTLER, D. What every equine practicioner should
Key words: Podiatry. Measurements. Equine. know about hoof balance .In: AMERICAN ASSOCIATION OF EQUINE PRACTITIONERS ANNUAL CONVENTION; 40, 1994, Vancouver, Proceedings... Vancouver: AAEP, 1995. p. 133-135.

3 TURNER, T. Hoof balance assessment and evaluation. In: ANNUALVETERINARIAN/FERRIER CONFERENCE,3. 1996, Saint Paul, Minnesota. Proceedings... p. 12-21.

4 KOBLUK C. et al. The Effect of conformation and 
shoeing: a cohort study of 95 thoroughbred racehorses. In: AMERICAN ASSOCIATION OF EQUINE PRACTITIONERS ANNUAL CONVENTION; 35, 1989. Boston. Proceedings... B oston: AAEP, 1990. p. 259-274.

5 SNOW, V. E. Specific parameters used to evaluate hoof balance and support. In: AMERICAN ASSOCIATION OF EQUINE PRACTITIONER ANNUAL CONVENTION, 38; 1992, Orlando. Proceedings... Orlando: AAEP, 1993. p. 299-311.

6 BALCH, O. et al. Hoof balance and lameness:improper toe length, hoof angle, and mediolateral balance. Compendium on Continuing Education for the Practicing Veterinarian, v. 17, p. 1275-1282, Oct. 1995.

7 NICOLETTI, J. L. M. et al. Mensuração do casco de eqüinos para identificação objetiva de anormalidades de conformação. Veterinária Notícias, Uberlândia, v. 6, n.1, p. 61-68, 2000

8 MURGA, J. A. Nutrición equilibrada, suplementación com Ferrier's fórmula y aplicación de antiséptico para cascos, em caballos pura raza espanhola com padecimiento de síndrome de talones contraídos (encastilladura). In: CONGRESSO NACIONAL DE MÉDICOS VETERINÁRIOS ESPECIALISTAS EM EQUINOS,21.; CONGRESSO IBEROAMERICANO,2.,1999, San Miguel Allende, México. Anais... San Miguel Allende, México, 1999. p. 85-87.

9 O'GRADY, S. E.; POUPARD, D. A. Physiological horseshoeing: an overview. Equine Veterinary Education, Lexington, v. 28, n. 4, p. 426-430,dez. 2001.

10 STASHAK, T. Adam's lameness in horses. 5 ed. Philadelphia: Lippincott Williams \&Wilkins, 2002. 